Japanese Journal of Applied Physics, 27 (1) (1988) 151-152

\title{
Fluorescence from Pearls of Freshwater Bivalves and Its Contribution to the Distinction of M other Oysters Used in Pearl Culture
}

Tadaki Miyoshi, Yasunori Matsuda ${ }^{1}$ and Shigeru Akamatsu ${ }^{1}$

Technical College, Yamaguchi U niversity, Tokiwadai, U be, Yamaguchi 755

${ }^{1}$ Pearl Research laboratory, K. M IKIM OTO \& Co., Ltd., Toba, M ie 517

(Received October 24, 1987; accepted November 28, 1987)

By measuring the fluorescence spectra of pearls from various species of mother oysters, a distinction has already become possible as to whether the pearls are from Pinctada fucata, Pinctada maxima, Pteria penguin or Pinctada margaritifera. In this study, the fluorescence of pearls from freshwater bivalves was measured. The results have made it possible to distinguish freshwater pearls from the other pearls mentioned above.

KEYWORDS: I aser-i nduced fI uor escence, pear I, freshwat er bi val ve, nondest ructi ve di st i ncti on 
In present-day pearl culturing Pinctada fucata (Akoya oyster) is the most common and important mother oyster. However, several kinds of mother oysters are also used: Pinctada maxima (yellow lip oyster), Pteria penguin (Mabe), Pinctada margaritifera (black lip oyster), Genus Haliotis (abalone) and Hyriopsis shlegeli (Ikecho). From the viewpoint of gem appraising, nondestructive methods for determining the parentage of pearls are very important.

As for the distinction between pearls from seawater and freshwater bivalves, it has been reported that the detection of the quantity of manganese using X-ray fluorescence analysis is effective, ${ }^{1)}$ since freshwater pearls contain far more manganese than the seawater type. ${ }^{2)}$ However, the disadvantage of this method of analysis is that samples become blackish in colour as a result of X-ray irradiation, though the degree is small. Therefore, X-ray fluorescence analysis is not a perfectly non-destructive method.

In previous papers, ${ }^{3-5)}$ we have discussed the fact that pearls of P. fucata, P. maxima, P. penguin and P. margaritifera can be distinguished nondestructively from each other by using various differences in the fluorescence and reflection spectra. In this study, we applied this fluorescence measurement technique in order to distinguish freshwater and seawater pearls.

The fluorescence spectra have been measured at room temperature with the following apparatus (similar to that described in ref. 4). The excitation source was a pulsed $\mathrm{N}_{2}$ laser (NDC JS-1000L, $\lambda=337.1 \mathrm{~nm}$, pulse duration $=5 \mathrm{~ns}$, repetition rate $=$ $4 \mathrm{~Hz}$ ). The laser beam was set at an angle of about $50^{\circ}$ off the normal incidence to the plane of the sample and focused on a spot of about $1 \mathrm{~mm}^{2}$ in area by a quartz lens. The peak intensity of the laser light on the sample was about $50 \mathrm{~kW} / \mathrm{cm}^{2}$. Fluorescence was observed at $90^{\circ}$ to the laser beam and was focused on the entrance slit of a $50 \mathrm{~cm}$ monochromator (Oyo Bunko ASI-50S) by a glass lens. The fluorescence spectra were measured with a monochromator, a photomultiplier (Hamamatsu R955), a boxcar integrator (homemade) and a recorder. Ordinary fluorescence spectra have been measured with a spectrofluorophotometer (Japan Spectroscopic FP-770). The apparatus constant was not corrected for the ordinary fluorescence spectra.

In this study, both Japanese and Chinese freshwater pearls were used as specimens. Hyriopsis shlegeli (Ikecho) is a mother oyster of Japanese pearl; however, the Chinese 
pearl mother oyster is not known. These samples have various colours: white, pink, orange, grey and brown. For the purpose of a comparison, pearls of seawater bivalves such as P. fucata, P. maxima, P. penguin and P. margaritifera were also used as fluorescence measuring specimens.

Flgure 1 shows the fluorescence spectra of white pearls of Hyriopsis shlegeli, P. fucata, P. maxima, P. penguin and P. margaritifera. The pearl of Hyriopsis shlegeli shows a peak at about $410 \mathrm{~nm}$, while pearls of other mother oysters have peaks at wavelengths longer than $430 \mathrm{~nm}$. The fluorescence spectra of Chinese freshwater pearls are almost the same as those of Hyriopsis shlegeli. Thus, this difference in the fluorescence spectra makes it possible to distinguish among pearls of different origin. ${ }^{*}$

It seems likely that the peak wavelength of fluorescence is correlated with the species of the sample pearls. In order to examine this relationship, the peak wavelengths were measured with white pearls of freshwater bivalves, P. fucata and P. maxima. Figure 2 shows the peak-wavelength distribution of these pearls: pearls of freshwater bivalves (dotted lines), P. maxima (dashed lines) and P. fucata (solid lines). As is shown, the peak wavelengths of these three do not overlap each other, which makes a distinction possible for three different kinds of white pearls. Pearls of $P$. penguin and $P$. margaritifera do not appear in Fig. 2, since these pearls can be distinguished from other pearls using a peculiar fluorescence peak at $620 \mathrm{~nm}$ under $400 \mathrm{~nm}$ light excitation. ${ }^{4,5}$

Freshwater pearls with other colours were also measured. Pink, orange and grey pearls show similar spectra to those of white pearls. On the contrary, brown pearls show similar spectra to those of P. maxima with a peak at $430-440 \mathrm{~nm}$. Therefore, this result makes distinction difficult for this colour.

In summary, the fluorescence spectra of pearls of different mother oysters have been measured. The difference in peak wavelength makes it possible to distinguish pearls of freshwater bivalves from those of P. fucata, P. maxima, P. penguin and P. margaritifera, with only one exception: brown freshwater pearls.

*Ordinary fluorescence spectra were measured at various excitation wavelengths: 320-380 $\mathrm{nm}$. The fluorescence spectra depend on the excitation wavelength. The 
fluorescence band could not be observed under $320 \mathrm{~nm}$ light excitation. On the other hand, the short-wavelength side of the fluorescence band overlapped with the excitation light under long-wavelength light excitation. Thus, $350 \mathrm{~nm}$ light excitation is suitable for fluorescence measurements. The fluorescence spectra of several pearls under 350 $\mathrm{nm}$ light excitation are similar to those under $\mathrm{N}_{2}$ laser excitation $(\lambda=337.1 \mathrm{~nm})$. 
R eferences

1) K. Wada: Shinju (Pearl) (Zenkoku Hosekigaku Kyokai, Tokyo, 1982) p. 198 [in Japanese].

2) Y. Horiguchi: Bull. Jpn. Soc. Sci. Fish. 25 (1959) 392 [in Japanese].

3) T. Miyoshi, Y. Matsuda and H. Komatsu: Jpn. J. Appl. Phys. 25 (1986) 1606.

4) T. Miyoshi, Y. Matsuda and H. Komatsu: Jpn. J. Appl. Phys. 26 (1987) 578.

5) T. Miyoshi, Y. Matsuda and H. Komatsu: Jpn. J. Appl. Phys. 26 (1987) 1069. 
Figure captions

Fig. 1. Fluorescence spectra of pearls of several mother oysters at $300 \mathrm{~K}$ under $\mathrm{N}_{2}$ laser excitation. Peak intensities are normalized.

Fig. 2. Distribution of the peak wavelengths of fluorescence from white pearls of freshwater bivalves (Hyriopsis shlegeli et al.) (dotted lines) , P. maxima (dashed lines) and P. fucata (solid lines). 
Fig. 1

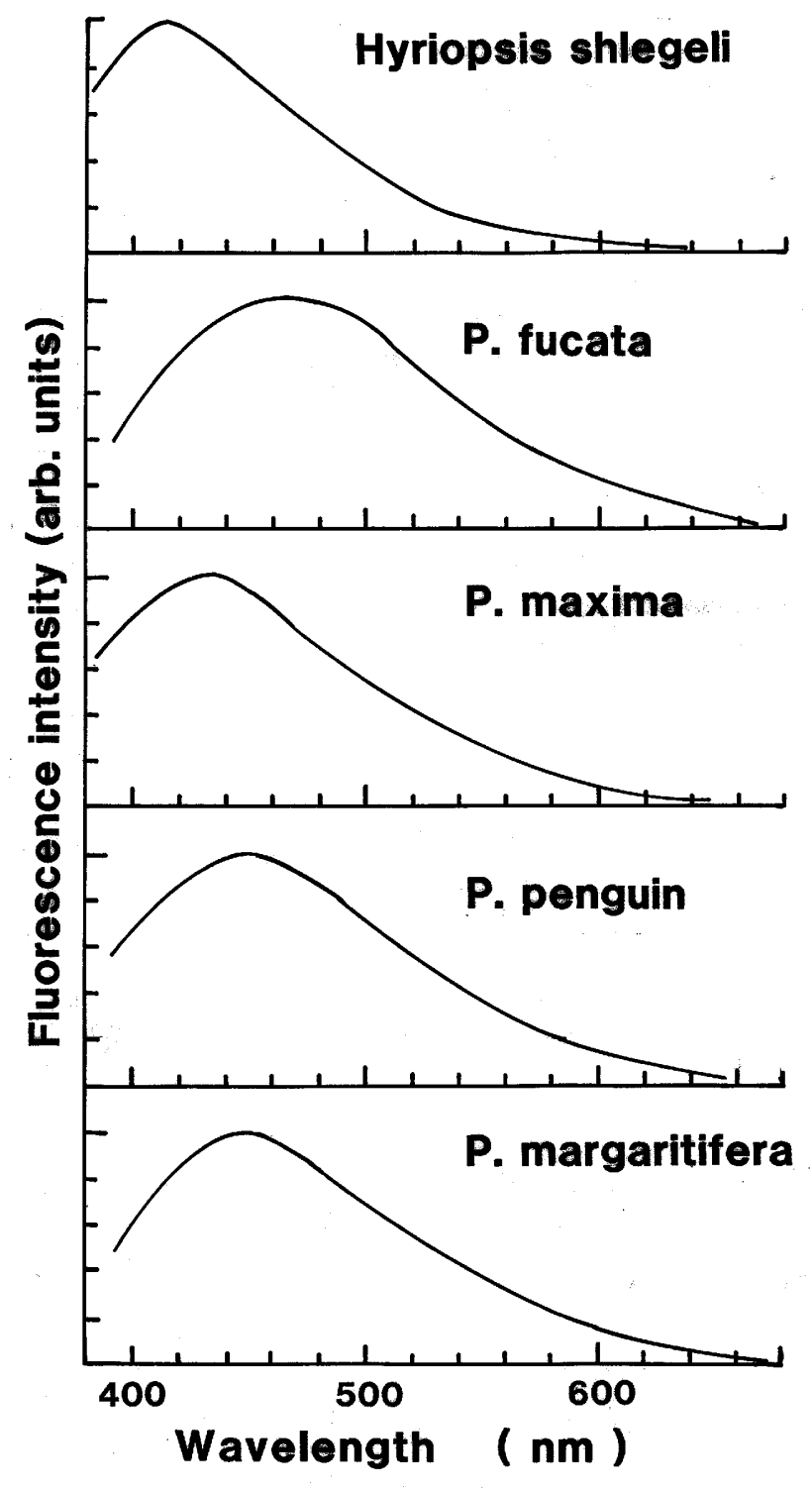

Fig. 2

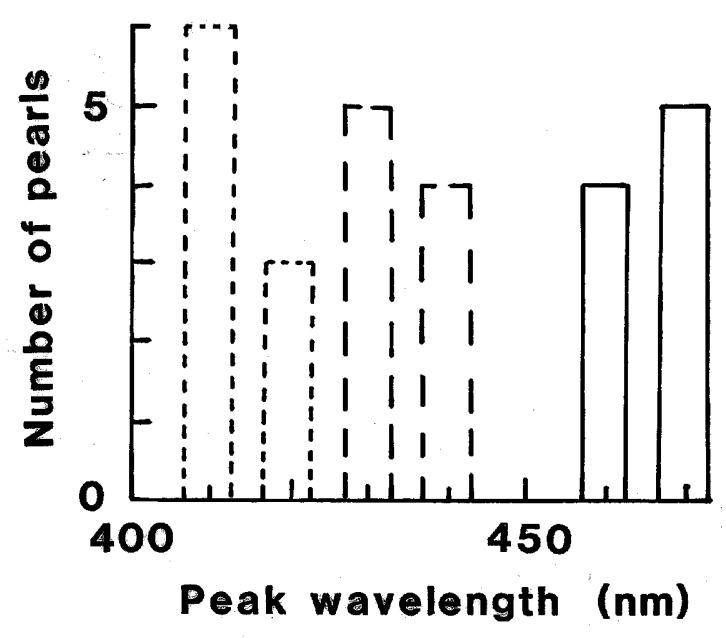

